\title{
Editorial: Terahertz Sensing and Diagnosis
}

\author{
Yi Huang ${ }^{1}$, Shuncong Zhong ${ }^{1 *}$ and Yao-chun Shen ${ }^{2}$ \\ ${ }^{1}$ Laboratory of Optics, Terahertz and Non-Destructive Testing, School of Mechanical Engineering and Automation, Fuzhou \\ University, Fuzhou, China, ${ }^{2}$ Department of Electrical Engineering and Electronics, University of Liverpool, Liverpool, \\ United Kingdom
}

Keywords: terahertz non-destructive testing, sensing, diagnosis, metamaterials, plasmonic surface, resonant electricfield enhancement

\section{Editorial on the Research Topic}

\section{Terahertz Sensing and Diagnosis}

As a transition frequency region from macroscopic electronics to microscopic photonics, terahertz $(\mathrm{THz})$ radiation has attracted great attention from the scientists. But for a long time, due to the lack of effective $\mathrm{THz}$ radiation generation and detection methods, the electromagnetic waves in the terahertz band have not been studied and applied, so this part of the electromagnetic spectrum has been name the "THz gap" [1]. In fact, as early as 100 years ago, there were scientists that studied electromagnetism in this frequency region. Between 1896 and 1897, Rubens and Nichols made an initial exploration of this frequency band [2]. In the following hundred years, some technical developments and many relevant theories have been elaboratoed. Even, few $\mathrm{THz}$ devices were reported [3, 4]. Yet, the word “Terahertz" first appeared in 1974, when Fleming used it to describe a spectrum line covered by the Michelson interferometer [1]. The rapid development of modern $\mathrm{THz}$ science and technology came in the mid-1980s, with the development of a series of new technologies and materials. This was due to the development of ultra-fast laser technology, making it a routine technology to obtain a stable broadband pulse THz source. In recent years, with the development and updating of $\mathrm{THz}$ source and detection technology, and the great expansion of application fields, the research and application of $\mathrm{THz}$ technology has undergone extensive and profound changes.

The $\mathrm{THz}$ frequency region is especially suitable for local area network and broadband mobile communication because of the high frequency, wide bandwidth and multi-channel characteristics of $\mathrm{THz}$ wave. Thus, it has been considered as a key technology of 6G. An ultra-high wireless transmission speed that is hundreds or even thousands of times faster than current ultra-wideband technology can be achieved by using $\mathrm{THz}$ waves to communicate [5]. Moreover, since the interstellar radiation is mostly in the submillimeter wavelengths, namely $\mathrm{THz}$ band, its $\mathrm{THz}$ spectroscopy contains the rich information of the star formation process and the interstellar medium chemical properties. Therefore, more interesting astronomical observation can be acquired by using $\mathrm{THz}$ detection technology. In 2019, astronomers around the world, including in China, simultaneously released the first photo of a black hole in human history, which was directly taken by the $\mathrm{THz}$ telescope. Furthermore, due to the low radiation power and the strong penetration of $\mathrm{THz}$ radiation, it can replace X-ray fluoroscopy and computed tomography scan in anti-terrorism security check and non-destructive testing. In 2020, due to the impact of the COVID-19 pandemic, all passengers have to first check the temperature and then go through security at every major transportation hubs, which is complicated and inefficient, greatly increasing the chance of virus infection. But, the temperature detection and security check can be simultaneously completed in just a few seconds without contact at transportation hubs equipped with $\mathrm{THz}$ security apparatus, which greatly improves passage efficiency.

In addition to high-speed communications, astronomy, and anti-terrorism security, the focus of $\mathrm{THz}$ research has shifted to non-destructive testing (NDT) applications due to its unique 
electromagnetic characteristics. Since the vibrational and rotational frequencies of most organic molecules and biological macromolecules are in $\mathrm{THz}$ range, and since the $\mathrm{THz}$ pulses have a good temporal resolution (picosecond magnitude), terahertz time-domain spectroscopy (THz-TDS) has been widely used in NDT. In the last 10 years or so, this has led to remarkable progress in the understanding of THz-NDT with the potential for huge impact on a wide range of applications, from biomedical sensing to engineering diagnosis $[6,7]$. This also resulted in the development of innovative $\mathrm{THz}$ technologies that provide biosensing, and precise diagnostics with unprecedented ultrahigh sensitivity, resolution, and detection speed [8]. However, the absorption cross-sections of molecules are extremely small when compared to the $\mathrm{THz}$ wavelengths, leading to rather weak interactions between molecules and $\mathrm{THz}$ radiation [9]. For this reason, it is difficult to perform selective sensing or quantiative analysis of substances based on their $\mathrm{THz}$ characteristic responses. Enhancing the sensing sensitivity, detection range, detection speed, and resolution of $\mathrm{THz}$ spectroscopy technology, and quickly and conveniently realizing the detection of small amount of samples have become urgent issues to be addressed. Luckily, today's electromagnetic plasmonic surface technology can provide a solution to these problems due to its unique resonant electric field enhancement effect.

This Research Topic was designed to highlight the recent progresses and trends in the development of leading-edge terahertz NDT technologies, including sensing and diagnosis, and serve as a reference for the community. It contains several "Original Research" articles. A major focus is on the research associated with the utilization of terahertz metamaterial for sensing applications. These articles include a discussion of the

\section{REFERENCES}

1. Siegel PH. Terahertz Technology. IEEE Trans Microwave Theor Techn. (2002) 50(3):910-28. doi:10.1109/22.989974

2. Rubens H, and Snow BW. II. On the Refraction of Rays of Great Wave-Length in Rock-Salt, Sylvite, and Fluorite. Lond Edinb Dublin Philosophical Mag J Sci (1893) 35(212):35-45. doi:10.1080/14786449308620376

3. Kimmitt MF. Restrahlen to T-Rays - 100 Years of Terahertz Radiation. J Biol Phys (2003) 29(2-3):77-85. doi:10.1023/A:1024498003492

4. Wiltse JC. History of Millimeter and Submillimeter Waves. IEEE Trans Microwave Theor Techn. (1984) 32(9):1118-27. doi:10.1109/TMTT.1984.1132823

5. Jornet JM, and Akyildiz IF. Femtosecond-Long Pulse-Based Modulation for Terahertz Band Communication in Nanonetworks. IEEE Trans Commun (2014) 62(5):1742-54. doi:10.1109/TCOMM.2014.033014.130403

6. Zhong S. Progress in Terahertz Nondestructive Testing: A Review. Front Mech Eng (2019) 14(3):273-81. doi:10.1007/s11465-018-0495-9

7. Huang Y, Zhong S, Shi T, Shen Y-c., and Cui D. Terahertz Plasmonic PhaseJump Manipulator for Liquid Sensing. Nanophotonics (2020) 9(9):3011-21. doi:10.1515/nanoph-2020-0247

8. Zhang H, Zhang P, Zhao J, Liu Y, Huang Y, Huang H, et al. The Hole-Tunneling Heterojunction of Hematite-Based Photoanodes Accelerates Photosynthetic sensing enhancement due to the electromagnetically induced transparency effect in terahertz metamaterial by substrate etching (Lin et al.), an ultrasensitive $\mathrm{THz}$ sensor based on centrosymmetric F-shaped metamaterial resonators (Ma et al.), and a three-dimensional dual-band terahertz perfect absorber for measuring the refractive index of analytes (Yin et al.). The Research Topic also involves several "Original Research" articles of $\mathrm{THz}$ monitoring and diagnosis. A research on destructive and non-destructive crop water status monitoring and diagnosis by terahertz imaging is authored by ( $\mathrm{Li}$ et al.). The capability of identifying different volatile liquids of two terahertz systems (i.e., $100 \mathrm{GHz}$ sub-THz line scanner and attenuation total reflection-based THz-TD) is demonstrated by Baxter et al., while a non-destructive evaluation method for hidden defects beneath the multilayer organic protective coatings using terahertz pulse imaging technology is presented by ( $\mathrm{Tu}$ et al.).

\section{AUTHOR CONTRIBUTIONS}

All authors listed have made a substantial, direct and intellectual contribution to the work, and approved it for publication. In detail, YH wrote the initial draft. YS gave comments, and SZ took the final look and modified.

\section{FUNDING}

National Natural Science Foundation of China (51675103); Fujian Provincial Science and Technology Project (2019I0004); State Key Laboratory of Mechanical System and Vibration (MSV2018-07); Shanghai Natural Science Fund (18ZR1414200).

Reaction. Angew Chem Int Ed (2021) 60(29):16009-18. doi:10.1002/ anie. 202102983

9. Huang Y, Zhong S, Shi T, Shen Y-C, and Cui D. HR-si Prism Coupled Tightly Confined Spoof Surface Plasmon Polaritons Mode for Terahertz Sensing. Opt Express (2019) 27(23):34067-78. doi:10.1364/OE.27.034067

Conflict of Interest: The authors declare that the research was conducted in the absence of any commercial or financial relationships that could be construed as a potential conflict of interest.

Publisher's Note: All claims expressed in this article are solely those of the authors and do not necessarily represent those of their affiliated organizations, or those of the publisher, the editors and the reviewers. Any product that may be evaluated in this article, or claim that may be made by its manufacturer, is not guaranteed or endorsed by the publisher.

Copyright (c) 2021 Huang, Zhong and Shen. This is an open-access article distributed under the terms of the Creative Commons Attribution License (CC BY). The use, distribution or reproduction in other forums is permitted, provided the original author(s) and the copyright owner(s) are credited and that the original publication in this journal is cited, in accordance with accepted academic practice. No use, distribution or reproduction is permitted which does not comply with these terms. 